\title{
Optimal Betti numbers of forest ideals
}

\author{
Michael Goff \\ Department of Mathematics \\ University of Washington, Seattle, WA 98195-4350, USA \\ mgof $f$ math. washington. edu
}

Submitted: Dec 19, 2008; Accepted: Mar 3, 2009; Published: Mar 13, 2009

Mathematics Subject Classification: 05E99, $13 \mathrm{D} 02$

\begin{abstract}
We prove a tight lower bound on the algebraic Betti numbers of tree and forest ideals and an upper bound on certain graded Betti numbers of squarefree monomial ideals.
\end{abstract}

\section{Introduction}

In this paper we consider bounds on the algebraic Betti numbers of squarefree monomial ideals. These ideals are naturally related both to hypergraphs and to simplicial complexes, and understanding the structure of their minimal free resolutions leads to insights into the combinatorics of hypergraphs and simplicial complexes. For example, the $f$-vector of a simplicial complex can be expressed as alternating sums of certain algebraic Betti numbers.

Several other papers, including [7], [10], and the survey paper [12], use combinatorial methods to describe the minimal free resolutions of edge ideals and to bound their Betti numbers. For example, Ferrers ideals, as described in [4] and [5], are conjectured in [17] and shown in [9] to minimize Betti numbers among edge ideals of bipartite graphs. Earlier papers construct bounds on Betti numbers in terms of the projective dimension [2] or the Hilbert function [1].

In general, while constructing explicit (generally nonminimal) resolutions such as the Taylor resolution is effective in finding upper bounds on Betti numbers, there are no standard techniques for finding lower bounds. One important lower bound on the Betti numbers is the Buchsbaum-Eisenbud-Horrocks conjecture, which states that for a graded module with projective dimension $l$ and Krull dimension 0, the $i$-th Betti number is at least $\beta_{i}(M) \geq\left(\begin{array}{l}l \\ i\end{array}\right)$. This was proven in [3] for a class of modules that includes all finite length quotients of $\mathrm{S}$ by monomial ideals, and [8] proves a version of this conjecture for modules of monomial type over local rings. In this paper we establish a tight lower 
bound for a class of squarefree monomial ideals known as forest ideals. We hope that our techniques can be used for other classes of ideals as well.

We start by reviewing the necessary background and introducing our notation. By $\mathbf{k}$ we always denote an arbitrary field, and $S$ is the polynomial ring over $\mathbf{k}$ in variables $V=\left\{x_{1}, \ldots, x_{n}\right\}$ with the usual $\mathbb{Z}$-grading. For a squarefree monomial ideal $I \subset S$, we consider the minimal free $\mathbb{Z}$-graded resolution:

$$
0 \rightarrow \bigoplus_{a \in \mathbb{Z}} S(-a)^{\beta_{l, a}} \rightarrow \ldots \rightarrow \bigoplus_{a \in \mathbb{Z}} S(-a)^{\beta_{0, a}} \rightarrow I \rightarrow 0
$$

In the above expression, $S(-a)$ denotes $S$ with grading shifted by $a$, and $l$ denotes the length of the resolution. In particular, $l \geq \operatorname{codim}(S / I)$. The numbers $\beta_{i, a}=\beta_{i, a}(I)$ are called the $\mathbb{Z}$-graded Betti numbers of $I$. We also consider the ungraded Betti numbers $\beta_{i}=\beta_{i}(I):=\sum_{a \in \mathbb{Z}} \beta_{i, a}(I)$.

Squarefree monomial ideals are closely related to hypergraphs by the edge ideal construction. A hypergraph $G=\left(V=\left\{x_{1}, \ldots, x_{n}\right\}, E\right)$ is a vertex set $V$ and a set of edges $E \subset 2^{V}$ with the property that no edge is contained in another edge. Note that we allow edges to have cardinality one, and we allow vertices that are not contained in an edge. The degree of $G$ is the maximum size of an edge. A hypergraph is pure if all its edges have the same cardinality. The edge ideal of $G$ is the ideal of $S$ given by

$$
I(G):=\left(x_{i_{1}} \ldots x_{i_{r}}:\left\{x_{i_{1}}, \ldots, x_{i_{r}}\right\} \in E\right) .
$$

Since each squarefree monomial ideal $I$ has a unique set of minimal generators, there exists a unique hypergraph $G_{I}$ whose edge ideal is $I$. Edge ideals were first introduced in [21]; results related to edge ideals can be found in [11], [13], [14], and [15].

The outline of the paper is as follows. We introduce our notation and definitions in Section 2. In Section 3, we prove a lower bound on the (ungraded) Betti numbers of hypertree ideals. For the edge ideal of a degree $d$ hypertree with a $d$-coloring and $n_{i}$ vertices of color $i, 1 \leq i \leq d$, this lower bound is given by

$$
\beta_{j-1}(I) \geq \sum_{i=1}^{d}\left(\begin{array}{c}
n_{i} \\
j
\end{array}\right) \text {. }
$$

We also prove an extension of that bound to hyperforests. Furthermore, we prove that for ordinary trees $(d=2)$, the bound is attained if and only if the tree has diameter at most 4. In Section 4, we look at upper bounds on the graded Betti numbers of squarefree monomial ideals and prove that for a degree $d$ ideal $I$ with $t$ minimal generators,

$$
\beta_{j-1, j d-1}(I) \leq\left(\begin{array}{c}
t \\
j
\end{array}\right)-\left(\begin{array}{c}
t_{1} \\
j
\end{array}\right)-\ldots-\left(\begin{array}{c}
t_{j-1} \\
j
\end{array}\right),
$$

where $\left(t_{1}, \ldots, t_{j-1}\right)$ is the partition of $t$ that maximizes the upper bound. Furthermore, this bound is tight when $j=3$. We also consider a related conjecture. We use the Taylor resolution for the proof of the upper bound. The proof of the lower bound requires techniques such as the Mayer-Vietoris sequence from algebraic topology. The proofs of both bounds make use Hochster's formula. Similar methods were used in [9]. 


\section{Preliminaries}

In this section we introduce our definitions and notations, and we review some standard results. A hyperforest is a hypergraph $G=(V, E)$ with the property that the edges of $G$ can be enumerated $F_{1}, \ldots, F_{|E|}$ in such a way that for all $2 \leq i \leq|E|, F_{i} \cap\left(F_{1} \cup \cdots \cup F_{i-1}\right) \subset$ $F_{j}$ for some $j<i$. $G$ is called a hypertree if the following conditions hold: $G$ is pure, every vertex of $G$ is contained in an edge, and the edges of $G$ can be enumerated so that for all $2 \leq i \leq|E|, F_{i} \cap\left(F_{1} \cup \cdots \cup F_{i-1}\right) \subset F_{j}$ for some $j<i$ and additionally $\left|F_{i}-\left(F_{1} \cup \cdots \cup F_{i-1}\right)\right|=1$. This definition is very different from the definition of a tree in [6]. If $G$ is pure and has degree 2 , then hyperforests and hypertrees are ordinary graphtheoretic forests and trees. The hypergraph with edges $\left\{x_{1} x_{2} x_{3}, x_{1} x_{2} x_{4}, x_{1} x_{2} x_{5}, x_{2} x_{4} x_{6}\right\}$ is a hypertree, the hypergraph with edges $\left\{x_{1} x_{2} x_{3}, x_{1} x_{2} x_{4}, x_{4} x_{5} x_{6}\right\}$ is a hyperforest but not a hypertree, and the hypergraph with edges $\left\{x_{1} x_{2} x_{3}, x_{3} x_{4} x_{5}, x_{2} x_{4} x_{6}\right\}$ is not a hyperforest.

We say that a hypergraph $G=(V, E)$ is $k$-colorable if there exists a function $\kappa: V \rightarrow$ $[k]$, called a $k$-coloring, such that no two vertices with the same $\kappa$-value belong to the same face. All degree $d$ hyperforests are $d$-colorable. Furthermore, all degree $d$ hypertrees have a unique $d$-coloring up to permutation of the colors.

We also use the notion of a simplicial complex. A simplicial complex $\Gamma$ with the vertex set $V$ is a collection of subsets of $2^{V}$ called faces such that $\Gamma$ is closed under inclusion. Contrary to the more standard definition of a simplicial complex, we do not insist that the singleton subsets of $V$ are faces. With every simplicial complex $\Gamma$ we associate its StanleyReisner ideal $I_{\Gamma} \subset S$ generated by the non-faces of $\Gamma: I_{\Gamma}:=\left(\prod_{x_{i} \in L} x_{i}: L \subset V, L \notin \Gamma\right)$ (see [20]). Likewise, given a squarefree monomial ideal $I \subset S$, we denote by $\Gamma(I)$ the simplicial complex $\Gamma$ on $V$ whose Stanley-Reisner ideal is $I$.

If $W \subset V$, then the induced subcomplex of $\Gamma$ on $W$, denoted $\Gamma[W]$, is the simplicial complex with vertex set $W$ and faces $\{F \in \Gamma: F \subset W\}$. If $v \in V$ and $\{v\}$ is a face in $\Gamma$, then the link of $v$, denoted $\mathrm{lk}_{\Gamma}(v)$, is the simplicial complex that has vertex set $V-\{v\}$ and faces $\{F-\{v\}: v \in F \in \Gamma\}$. The antistar of $v$ is $\Gamma-v:=\Gamma[V-\{v\}]$. Let $\tilde{\beta}_{p}(\Gamma):=\operatorname{dim}_{\mathbf{k}}\left(\tilde{H}_{p}(\Gamma ; \mathbf{k})\right)$ be the dimension of the $p$-th reduced simplicial homology of $\Gamma$ with coefficients in $\mathbf{k}$.

We make frequent use of Hochster's formula (see [20, Theorem II.4.8]), which states that: for $W \subset V$,

$$
\beta_{i, a}\left(I_{\Gamma}\right)=\sum_{W \subset V,|W|=a} \tilde{\beta}_{|W|-i-2}(\Gamma[W]) .
$$

The ungraded version of Hochster's formula states that

$$
\beta_{i}\left(I_{\Gamma}\right)=\sum_{W \subset V} \tilde{\beta}_{|W|-i-2}(\Gamma[W])
$$

One advantage of using simplicial complexes is that Mayer-Vietoris sequences, together with Hochster's formula, allow us to construct bounds on the Betti numbers of the corresponding squarefree monomial ideal.

Simplicial complexes and hypergraphs can be related via the Stanley-Reisner ideal. To a simplicial complex $\Gamma$, we associate a hypergraph $G_{\Gamma}:=G_{I_{\Gamma}}$. Likewise, to a hypergraph 
$G$, we associate the simplicial complex $\Gamma(G)=\Gamma(I(G))$. Thus the edges of $G$ are the minimal nonfaces of $\Gamma(G)$. Also, $G_{\Gamma(\tilde{G})}=\tilde{G}$ and $\Gamma\left(G_{\tilde{\Gamma}}\right)=\tilde{\Gamma}$.

If in a hypergraph $G$, a vertex $v$ is not contained in any edge, then equivalently $v$ is contained in every maximal face in $\Gamma:=\Gamma(G)$. In this case we say that $\Gamma$ is a cone with apex $v$ and write $\Gamma=\{v\} * \Gamma[V-\{v\}]$. All cones are acyclic; $\Gamma$ is called acyclic if all its reduced Betti numbers vanish.

We use the following well-known fact in some of our proofs.

Lemma 2.1 Let $\Gamma$ be a simplicial complex, and suppose that $\Gamma-v$ is acyclic for some $v \in V(\Gamma)$. Then $\tilde{\beta}_{p}(\Gamma)=\tilde{\beta}_{p-1}\left(\mathrm{lk}_{\Gamma}(v)\right)$ for all $p$.

Proof: $\quad$ Observe that $\Gamma=(\Gamma-v) \cup\left(\{v\} * \mathrm{lk}_{\Gamma}(v)\right)$ and that $\mathrm{lk}_{\Gamma}(v)=(\Gamma-v) \cap\left(\{v\} * \mathrm{k}_{\Gamma}(v)\right)$. The lemma follows from the portion of the Mayer-Vietoris sequence

$$
\tilde{H}_{p}(\Gamma-v ; \mathbf{k}) \rightarrow \tilde{H}_{p}(\Gamma ; \mathbf{k}) \rightarrow \tilde{H}_{p-1}\left(\mathrm{lk}_{\Gamma}(v) ; \mathbf{k}\right) \rightarrow \tilde{H}_{p-1}(\Gamma-v ; \mathbf{k}) .
$$

We can describe the operation of taking the link of a vertex on the level of hypergraphs. If $v$ is a vertex of $G=(V, E)$ and $\{v\} \notin E$, then define

$$
\operatorname{lk}_{G}(v):=G_{\mathrm{lk}_{\Gamma(G)}(v)} .
$$

Equivalently, to construct $\operatorname{lk}_{G}(v)$, remove $v$ from $V$, and for all edges $F$ of $G$ that contain $v$, replace $F$ by $F-\{v\}$; then delete any edges that become nonminimal under inclusion. Similarly, we define the antistar $G-v$ on the level of hypergraphs by $G_{\Gamma(G)-v}$. Equivalently, $G-v$ has vertex set $V-\{v\}$ and edge set consisting of all edges of $G$ that do not contain $v$. Note that $G-v$ might contain an isolated vertex (that is, a vertex not contained in any edge) even if $G$ does not. We also define the induced hypergraph on $W \subset V$ by $G[W]$; $G[W]$ has vertex set $W$ and edges $\{F: F \in E, F \subset W\}$.

We also use the Taylor resolution of a squarefree monomial ideal, which in general is not minimal. Suppose $I$ is the edge ideal of the hypergraph $G=(V, E)$ with $r$ edges. For each $\left\{x_{j_{1}}, \ldots, x_{j_{t}}\right\}=F_{j} \in E$, let $\mu_{j}$ be the monomial $x_{j_{1}} \cdots x_{j_{t}}$. The Taylor resolution is a cellular resolution, in the sense of [16], supported on the labeled simplex with $r$ vertices, labeled $\mu_{j}, 1 \leq j \leq r$. For more information on cellular resolutions, see Chapter 4 of [16]. In particular, the $\mathbb{Z}$-graded Betti numbers of the Taylor resolution are

$$
\beta_{(i-1), j}^{T}(I)=\left|\left\{W \subseteq[r]:|W|=i, \operatorname{deg} \operatorname{lcm}_{k \in W} \mu_{k}=j\right\}\right|, \quad 1 \leq i \leq r .
$$

Here and throughout the paper, $[r]:=\{1,2, \ldots, r\}$.

Remark 2.2 Since the Taylor resolution is a free resolution of an ideal $I, \beta_{i, j}(I) \leq \beta_{i, j}^{T}(I)$ for all $i, j$. 


\section{Betti numbers of forest ideals}

Our first main theorem establishes a lower bound on the Betti numbers of tree ideals. Recall the convention that $\left(\begin{array}{l}n \\ a\end{array}\right)=0$ if $a>n$.

Theorem 3.1 Let $G$ be a degree d hypertree on $n$ vertices with d-coloring $\kappa$, and suppose for $1 \leq i \leq d$, there are $n_{i}$ vertices of color $i$. Let $I$ be the edge ideal of $G$. Then for $j \geq 2$,

$$
\beta_{j-1}(I) \geq \sum_{i=1}^{d}\left(\begin{array}{c}
n_{i} \\
j
\end{array}\right)
$$

Proof: We use induction on $n$. In the case $n=d, G$ is a single edge, each $n_{i}=1$, and the result holds with $\beta_{j-1}(I)=0$ for $j \geq 2$. Consider $n>d$, and let $v$ be a leaf of $G$ (that is, a vertex contained in only one edge). Every hypertree has a leaf. Since $G-v$ is also a hypertree, the result holds for $I_{G-v}$ by the inductive hypothesis.

Suppose that $v$ is colored blue, and let $B \subset V$ be the set of blue vertices of $V$. To prove the result, we claim that for each $B^{\prime} \subseteq B$, there exists $U^{\prime} \subseteq V$ such that $U^{\prime} \cap B=B^{\prime}$, and

$$
\tilde{\beta}_{\left|U^{\prime}\right|-\left|B^{\prime}\right|-1}\left(\Gamma\left(G\left[U^{\prime}\right]\right)\right) \geq 1 .
$$

Assuming the claim, take all $B^{\prime}$ with $\left|B^{\prime}\right|=j$ and $v \in B^{\prime}$, and apply the ungraded form of Hochster's formula. This yields

$$
\begin{aligned}
& \beta_{j-1}(I(G))=\sum_{v \notin U} \tilde{\beta}_{|U|-j-1}(\Gamma(G[U]))+\sum_{v \in U} \tilde{\beta}_{|U|-j-1}(\Gamma(G[U])) \geq \\
& \beta_{j-1}(I(G-v))+\left(\begin{array}{c}
|B|-1 \\
j-1
\end{array}\right) .
\end{aligned}
$$

Here $\left(\begin{array}{c}|B|-1 \\ j-1\end{array}\right)$ is the number of ways to choose $j$ blue vertices from $V(G)$ when one of those vertices is $v$. This, together with $\left(\begin{array}{c}|B|-1 \\ j\end{array}\right)+\left(\begin{array}{c}|B|-1 \\ j-1\end{array}\right)=\left(\begin{array}{c}|B| \\ j\end{array}\right)$ proves the result.

Since $G$ is a hypertree, $G$ satisfies two conditions: Condition A is that no blue vertex in $G$ is isolated, and Condition B is that every edge contains exactly one blue vertex. Fix $B^{\prime}$ as above. Then $G\left[V-\left(B-B^{\prime}\right)\right]$ also satisfies Conditions A and B. We prove a statement stronger than the above claim: if $\tilde{G}$ is any colorable hypergraph with blue vertex set $B^{\prime}$ that satisfies Conditions $\mathrm{A}$ and $\mathrm{B}$, then there exists $U^{\prime} \subseteq V(\tilde{G})$ with $B^{\prime} \subseteq U^{\prime}$ and $\tilde{\beta}_{\left|U^{\prime}\right|-\left|B^{\prime}\right|-1}\left(\Gamma\left(\tilde{G}\left[U^{\prime}\right]\right)\right) \geq 1$. We use induction on $|V(\tilde{G})|$.

The base case is $V(\tilde{G})=B^{\prime}$. Then Conditions A and B imply that every vertex comprises a singleton edge, and that $\Gamma(\tilde{G})$ has no nonempty faces. Then $\tilde{\beta}_{-1}(\Gamma(\tilde{G}))=1$, and the claim holds.

Now suppose that there exists $u \in V(\tilde{G})-B^{\prime} . \quad \tilde{G}-u$ always satisfies Condition B. First consider the case that $\tilde{G}-u$ also satisfies Condition A. Then by the inductive hypothesis, there exists $U^{\prime} \subset V(\tilde{G}-u)$ such that $\tilde{\beta}_{\left|U^{\prime}\right|-\left|B^{\prime}\right|-1}\left(\Gamma\left((\tilde{G}-u)\left[U^{\prime}\right]\right)\right) \geq 1$, and so $\tilde{\beta}_{\left|U^{\prime}\right|-\left|B^{\prime}\right|-1}\left(\Gamma\left(\tilde{G}\left[U^{\prime}\right]\right)\right) \geq 1$ as well, proving the claim. 
Suppose then that $\tilde{G}-u$ does not satisfy Condition A. It follows from the construction of the link that $\mathrm{lk}_{\tilde{G}}(u)$ satisfies Condition B. We check that $\mathrm{lk}_{\tilde{G}}(u)$ satisfies Condition A. Consider a blue vertex $u_{r}$, which in $\tilde{G}$ is contained in the edge $F=\left\{u_{1}, \ldots, u_{r}\right\}$ since $\tilde{G}$ satisfies Condition A. We show that $u_{r}$ is contained in an edge in $\mathrm{lk}_{\tilde{G}}(u)$. If $u \in F$, then $F-\{u\}$ is an edge in $\operatorname{lk}_{\tilde{G}}(u)$ that contains $u_{r}$. Otherwise, $F$ is not an edge in $\operatorname{lk}_{\tilde{G}}(u)$ only if there exists an edge $F^{\prime}$ in $\tilde{G}$ with $u \in F^{\prime}$ and $F^{\prime}-\{u\} \subset F$. In this case, since $F^{\prime}-\{u\}$ contains a blue vertex and $F$ contains exactly one blue vertex, $u_{r} \in F^{\prime}-\{u\}$. Since $F^{\prime}-\{u\}$ is an edge in $\operatorname{lk}_{\tilde{G}}(u)$, we conclude that $u_{r}$ is contained in an edge in $\operatorname{lk}_{\tilde{G}}(u)$.

By the inductive hypothesis, there exists $U^{\prime \prime} \subset V(\tilde{G}-u)$ such that $B^{\prime} \subseteq U^{\prime \prime}$ and $\tilde{\beta}_{\left|U^{\prime \prime}\right|-\left|B^{\prime}\right|-1}\left(\Gamma\left(\operatorname{lk}_{\tilde{G}}(u)\left[U^{\prime \prime}\right]\right)\right) \geq 1$. Since $\tilde{G}-u$ does not satisfy Condition A, there exists a blue vertex $w$ such that $w$ is contained in no edge of $\tilde{G}-u$ and hence also in no edge of $\tilde{G}\left[U^{\prime \prime}\right]$. It follows that $\Gamma\left(\tilde{G}\left[U^{\prime \prime}\right]\right)$ is a cone and therefore acyclic. Then by Lemma 2.1,

$$
\tilde{\beta}_{\left|U^{\prime \prime}\right|-\left|B^{\prime}\right|}\left(\Gamma(G)\left[U^{\prime \prime} \cup\{u\}\right]\right)=\tilde{\beta}_{\left|U^{\prime \prime}\right|-\left|B^{\prime}\right|-1}\left(\operatorname{lk}_{\Gamma(\tilde{G})}(u)\left[U^{\prime \prime}\right]\right) \geq 1 .
$$

The claim follows by taking $U^{\prime}=U^{\prime \prime} \cup\{u\}$.

In the proof of Theorem 3.1, for a given $B^{\prime}$, there may be several sets $U^{\prime}$ such that $\tilde{\beta}_{\left|U^{\prime}\right|-\left|B^{\prime}\right|-1}\left(\Gamma\left(G\left[U^{\prime}\right]\right)\right) \geq 1$. In that case, the bound is a strict inequality.

Proposition 3.2 The lower bound in Theorem 3.1 can be attained.

Proof: We construct $G=(V, E)$ as follows. Label $V$ by $\left\{v_{1}, \ldots, v_{d}, U_{1}, \ldots, U_{d}\right\}$, where for each $1 \leq i \leq d, U_{i}=\left\{u_{i, 1}, \ldots, u_{i, n_{i}-1}\right\}$. Let $\left\{v_{1}, \ldots, v_{d}\right\} \in E$, and for all $1 \leq i \leq d$ and $1 \leq k \leq n_{i}-1$, let $\left\{v_{1}, \ldots, v_{i-1}, v_{i+1}, \ldots, v_{d}, u_{i, k}\right\} \in E$. Note that $G$ is a $d$-colorable hypertree with $n_{i}$ vertices of color $i$. We verify that $\beta_{j-1}(I(G))=\sum_{i=1}^{d}\left(\begin{array}{c}n_{i} \\ j\end{array}\right)$ for all $j \geq 2$ by considering all $W \subseteq V$ and applying Hochster's formula.

Consider $W \subseteq V$, and suppose $W$ satisfies the following conditions for some $1 \leq r \leq d$ : $W \cap U_{i}=\emptyset$ for $i \neq r, v_{i} \in W$ for $i \neq r$, and $W$ is not simply $\left\{v_{1} \ldots, v_{r-1}, v_{r+1}, \ldots, v_{d}\right\}$. Then $\tilde{\beta}_{d-2}(\Gamma(G)[W])=1$ and $\tilde{\beta}_{p}(\Gamma(G)[W])=0$ for $p \neq d-2$. This follows by repeated applications of Lemma 2.1 using each of the $v_{i}, i \neq r$.

Next we consider the case that $W$ does not satisfy these conditions. If $W \cap U_{i}=\emptyset$ for all $1 \leq i \leq d$ and $W \neq\left\{v_{1}, \ldots, v_{d}\right\}$, then $\Gamma(G)[W]$ is a simplex and hence acyclic unless $W=\emptyset$, in which case $\Gamma(G)[W]$ consists of only the empty set, and in that case $\tilde{\beta}_{-1}(\Gamma(G)[W])=1$ and $\tilde{\beta}_{p}(\Gamma(G)[W])=0$ for $p \neq-1$. Now suppose $u_{r, k} \in W$ and $v_{i} \notin W$ for some $1 \leq r \leq d, 1 \leq k \leq n_{r}-1$, and $i \neq r$. Then $\Gamma(G)[W]$ is a cone with apex $u_{r, k}$ and is therefore acyclic. Finally, suppose $u_{r, k}, u_{r^{\prime}, k^{\prime}} \in W$ for some $1 \leq r<r^{\prime} \leq d$, $1 \leq k \leq n_{r}-1$, and $1 \leq k^{\prime} \leq n_{r^{\prime}}-1$. Then $\Gamma(G)[W]$ is a cone with apex either $u_{r, k}$ or $u_{r^{\prime}, k^{\prime}}$ unless $v_{i} \in W$ for all $1 \leq i \leq d$. In that case, $\Gamma(G)[W]-v_{r}$ is a cone with apex $u_{r^{\prime}, k^{\prime}}$ and $\operatorname{lk}_{\Gamma(G)[W]}\left(v_{r}\right)$ is a cone with apex $u_{r, k}$, and so it follows from Lemma 2.1 that $\Gamma(G)[W]$ is acyclic.

By the ungraded form of Hochster's formula, $W=\emptyset$ or $W=\left\{v_{1}, \ldots, v_{d}\right\}$ do not contribute to the algebraic Betti numbers of $I(G)$. For $j \geq 2$, there are $\left(\begin{array}{c}n_{i}-1 \\ j\end{array}\right)$ sets $W$ of the form $\left\{v_{1}, \ldots, v_{i-1}, v_{i+1}, \ldots, v_{d}, \tilde{W}\right\}$ with $|\tilde{W}|=j, \tilde{W} \subseteq U_{i}$ that each contribute 1 to 
$\beta_{j-1}(I(G))$, and there are $\left(\begin{array}{c}n_{i}-1 \\ j-1\end{array}\right)$ sets $W$ of the form $\left\{v_{1}, \ldots, v_{d}, \tilde{W}\right\}$ with $|\tilde{W}|=j-1, \tilde{W} \subseteq$ $U_{i}$ that each contribute 1 to $\beta_{j-1}(I(G))$. We conclude that $\beta_{j-1}(I)=\sum_{i=1}^{d}\left(\begin{array}{c}n_{i} \\ j\end{array}\right)$.

In the case of degree 2 trees, we fully answer the question of equality. For two vertices $u$ and $v$ of a connected graph $G$, a path joining $u$ and $v$ is a set of vertices $u=u_{0}, u_{1}, \ldots, u_{l}=$ $v$ such that $u_{i-1} u_{i}$ is an edge for $1 \leq i \leq l$. Let dist $(u, v)$ denote the fewest number of edges in a path joining $u$ and $v$. The diameter of $G$ is $\max _{u, v}(\operatorname{dist}(u, v))$.

The proof of Theorem 3.3 uses the Künneth formula. Assume that $V$ is the disjoint union $V_{1} \sqcup V_{2}$. In this case, we say that $\Gamma=\Gamma[V]$ is the simplicial join $\Gamma\left[V_{1}\right] * \Gamma\left[V_{2}\right]$ if the edge set of $G_{\Gamma}$ is the disjoint union of the edges sets of $G_{\Gamma\left[V_{1}\right]}$ and $G_{\Gamma\left[V_{2}\right]}$. For such $\Gamma$, the Künneth formula (see [20, Section 5.3]) states that

$$
\tilde{\beta}_{j-1}(\Gamma)=\sum_{r+s=j} \tilde{\beta}_{r-1}\left(\Gamma\left[V_{1}\right]\right) \tilde{\beta}_{s-1}\left(\Gamma\left[V_{2}\right]\right) .
$$

Theorem 3.3 Let $G=(V, E)$ be a colored tree with blue vertex set $B$ and red vertex set $R$, with $|B|=n_{1}$, and $|R|=n_{2}$. Then $\beta_{j-1}(I(G))=\left(\begin{array}{c}n_{1} \\ j\end{array}\right)+\left(\begin{array}{c}n_{2} \\ j\end{array}\right)$ for all $j \geq 2$ if and only if $G$ has diameter at most four.

Proof: First suppose $G$ has diameter greater than four. Then $G$ has an induced subtree $G^{\prime}$ that is a path on six vertices, of which three are red and three are blue. One can check that $\beta_{1}\left(I\left(G^{\prime}\right)\right)=7>\left(\begin{array}{l}3 \\ 2\end{array}\right)+\left(\begin{array}{l}3 \\ 2\end{array}\right)$. It follows by induction, as in the proof of Theorem 3.1, that $\beta_{1}(I(G))>\left(\begin{array}{c}n_{1} \\ 2\end{array}\right)+\left(\begin{array}{c}n_{2} \\ 2\end{array}\right)$.

Now suppose $G$ has diameter at most four. There exists $v \in V$ such that for all $u \in V$, $\operatorname{dist}(u, v) \leq 2$. Assume without loss of generality that $v$ is blue. If dist $(u, v)=1$, then $u$ is red, while if dist $(u, v)=2$, then $u$ is blue. Furthermore, all blue vertices except $v$ are leaves. For each blue vertex $u \neq v$, let $p(u)$ be the unique neighbor of $u$. Set $\Gamma=\Gamma(G)$. We show that $\beta_{j-1}(I(G))=\left(\begin{array}{c}n_{1} \\ j\end{array}\right)+\left(\begin{array}{c}n_{2} \\ j\end{array}\right)$ for all $j \geq 2$ by considering the induced subcomplex on $\Gamma[W], W \subseteq V$ in several cases and applying the ungraded form of Hochster's formula. Every $W \subseteq V$ is covered by exactly one of the following cases.

Case 1: $v \notin W$. For all blue $u \in W, \Gamma[W]$ is a cone with apex $u$ and therefore acyclic unless $p(u) \in W$. Also, if $w \in W$ is a red vertex and $w \neq p(u)$ for any blue $u \in W$, then $\Gamma[W]$ is a cone with apex $w$. If $p(u) \in W$ for all blue $u \in W$, and all red vertices $w \in W$ satisfy $w=p(u)$ for some $u \in W$, then we claim that $\tilde{\beta}_{s-1}(\Gamma[W])=1$ and $\tilde{\beta}_{t}(\Gamma[W])=0$ for $t \neq s-1$, where $s=|W \cap R|$. Such a $W$ is uniquely determined by a subset of $B-\{v\}$, and therefore there are $\left(\begin{array}{c}n_{1}-1 \\ j\end{array}\right)$ such $W$ that, by Hochster's formula, each contribute 1 to $\beta_{j-1}(I(G))$.

The claim is true for $s=1$ since in that case, $\Gamma[W]$ is the disjoint union of a simplex and a vertex. For $s>1, \Gamma[W]$ is the simplicial join of $s$ such complexes, and the Künneth formula proves the claim.

Case 2: $W \cap B=\{v\}$ and $W \cap R \neq \emptyset$. Then $\tilde{\beta}_{0}(\Gamma[W])=1$ and $\tilde{\beta}_{j}(\Gamma[W])=0$ for $j \neq 0$, since in that case, $\Gamma[W]$ is the disjoint union of a vertex and a simplex. Such subsets $W$ can be indexed by nonempty subsets of $R$, and therefore there are $\left(\begin{array}{c}n_{2} \\ j\end{array}\right)$ such $W$ that, by 
Hochster's formula, each contribute 1 to $\beta_{j-1}(I(G))$. Note that if $W=\{v\}$, then $\Gamma[W]$ a single vertex and therefore acyclic.

Case 3: $\{v\} \subsetneq W \cap B$. Let $u \in W \cap B$ with $u \neq v$. Then $\Gamma[W]$ is a cone with apex $u$ unless $p(u) \in W$, so assume $p(u) \in W$. Consider $\operatorname{lk}_{\Gamma[W]}(p(u))$. Since $\Gamma[W]-p(u)$ is a cone and therefore acyclic, we apply Lemma 2.1 and conclude that $\tilde{\beta}_{t}(\Gamma[W])=\tilde{\beta}_{t-1}\left(\mathrm{lk}_{\Gamma[W]}(p(u))\right)$ for all $t$.

As in Case $1, \mathrm{lk}_{\Gamma[W]}(p(u))$ is a cone and therefore acyclic unless for every $w \in R \cap W$, $w=p(z)$ for some $z \in W \cap B$, and also for all $z \in W \cap B, p(z) \in W$. If that condition is also satisfied, then $\tilde{\beta}_{s-2}\left(\mathrm{lk}_{\Gamma}(p(u))[W]\right)=1$ and $\tilde{\beta}_{t}\left(\mathrm{lk}_{\Gamma}(p(u))[W]\right)=0$ for $t \neq s-2$, where $s=|W \cap R|$, by the reasoning of Case 1 . Therefore $\tilde{\beta}_{s-1}(\Gamma[W])=1$ and $\tilde{\beta}_{t}(\Gamma[W])=0$ for $t \neq s-1$. Such a $W$ is uniquely determined by a subset of $B-\{v\}$, and therefore there are $\left(\begin{array}{c}n_{1}-1 \\ j-1\end{array}\right)$ such $W$ that, by Hochster's formula, each contribute 1 to $\beta_{j-1}(I(G))$.

It follows from the three cases above that $\beta_{j-1}(I(G))=\left(\begin{array}{c}n_{1}-1 \\ j\end{array}\right)+\left(\begin{array}{c}n_{1}-1 \\ j-1\end{array}\right)+\left(\begin{array}{c}n_{2} \\ j\end{array}\right)=$ $\left(\begin{array}{c}n_{1} \\ j\end{array}\right)+\left(\begin{array}{c}n_{2} \\ j\end{array}\right)$ for all $j \geq 2$.

For the case $d>2$, we wonder if there is a simple combinatorial property that characterizes equality. We phrase this as a question.

Question 3.4 For a degree d hypertree ideal $I$, when is $\beta_{j-1}(I)=\sum_{i=1}^{d}\left(\begin{array}{c}n_{i} \\ j\end{array}\right)$ for all $j \geq 2$ ?

Theorem 3.1 can be used to establish a lower bound on the Betti numbers of forest ideals. If $r$ is an integer, we say that the sequence of integers $\left(r_{1}, \ldots, r_{d}\right)$ is a nearly even $d$-partition of $r$ if for all $1 \leq i<j \leq d,\left|r_{i}-r_{j}\right| \leq 1$.

Theorem 3.5 Let $G$ be a degree $d$ hyperforest with $t$ edges, and let $\left(n_{1}, \ldots, n_{d}\right)$ be a nearly even $d$-partition of $t+d-1$. Let $I$ be the edge ideal of $G$. Then for $j \geq 2$,

$$
\beta_{j-1}(I) \geq \sum_{i=1}^{d}\left(\begin{array}{c}
n_{i} \\
j
\end{array}\right)
$$

Proof: Enumerate the edges of $G$ by $F_{1}, \ldots, F_{t}$ in such a way that for $2 \leq i \leq t$, $\left|F_{i}-\left(F_{1} \cup \cdots \cup F_{i-1}\right)\right| \geq 1$. For $2 \leq k \leq t$, choose $v_{k} \in F_{k}-\left(F_{1} \cup \cdots \cup F_{k-1}\right)$. Let $\kappa$ be a $d$-coloring of $G$, and set $n_{i}^{\prime}=1+\left|\left\{k: 2 \leq k \leq t, \kappa\left(v_{k}\right)=i\right\}\right|$. First we show that for $j \geq 2, \beta_{j-1}(I) \geq \sum_{i=1}^{d}\left(\begin{array}{c}n_{i}^{\prime} \\ j\end{array}\right)$. We apply induction on $t$, with the case $t=1$ evident. Suppose without loss of generality that $\kappa\left(v_{t}\right)=1$. Then by the inductive hypothesis,

$$
\beta_{j-1}\left(I\left(G-\left(F-\left(F_{1} \cup \cdots \cup F_{k-1}\right)\right)\right)\right) \geq\left(\begin{array}{c}
n_{1}^{\prime}-1 \\
j
\end{array}\right)+\sum_{i=2}^{d}\left(\begin{array}{c}
n_{i}^{\prime} \\
j
\end{array}\right) .
$$

By taking $v_{t}$ to be a blue vertex, $G$ satisfies Conditions $\mathrm{A}$ and $\mathrm{B}$ as defined in the proof of Theorem 3.1, and so by the argument in that proof, $\beta_{j-1}(I(G)) \geq \sum_{i=1}^{d}\left(\begin{array}{c}n_{i}^{\prime} \\ j\end{array}\right)$.

To complete the proof, observe that among all partitions $\left\{n_{1}^{\prime}, \ldots, n_{d}^{\prime}\right\}$ of $t+d-1$, the sum $\sum_{i=1}^{d}\left(\begin{array}{c}n_{i}^{\prime} \\ j\end{array}\right)$ is minimized when $\left\{n_{1}^{\prime}, \ldots, n_{d}^{\prime}\right\}$ is nearly even.

The lower bound of Theorem 3.5 for $\beta_{j-1}(I)$ is approximately $d^{-j+1} \frac{t^{j}}{j !}$ for large $t$. This contrasts to an upper bound, following from the Taylor resolution, of approximately $\frac{t^{j}}{j !}$. 


\section{Upper Bounds on Graded Betti Numbers}

In this section we establish some upper bounds on the graded Betti numbers of squarefree monomial ideals.

A simple observation is that for a squarefree monomial ideal $I$ with $t$ generators, $\beta_{i-1, j}(I) \leq\left(\begin{array}{l}t \\ i\end{array}\right)$. This follows from the Taylor resolution. For a pure degree $d$ ideal, this bound can be improved for the Betti number $\beta_{j-1, j d-1}$.

Theorem 4.1 Let I be a pure degree d squarefree monomial ideal with $t$ generators, and let $\left(t_{1}, t_{2}, \ldots, t_{j-1}\right)$ be a nearly even $(j-1)$-partition of $t$. Then

$$
\beta_{j-1, j d-1}(I) \leq\left(\begin{array}{l}
t \\
j
\end{array}\right)-\left(\begin{array}{c}
t_{1} \\
j
\end{array}\right)-\ldots-\left(\begin{array}{c}
t_{j-1} \\
j
\end{array}\right) .
$$

Proof: We show, using the Taylor resolution, that

$$
\beta_{j-1, j d-1}^{T}(I) \leq\left(\begin{array}{c}
t \\
j
\end{array}\right)-\left(\begin{array}{c}
t_{1} \\
j
\end{array}\right)-\ldots-\left(\begin{array}{c}
t_{j-1} \\
j
\end{array}\right) .
$$

Suppose that $I$ is the edge ideal of a hypergraph $G=(V, E)$ with edges $\left\{F_{1}, \ldots, F_{t}\right\}$. Construct a graph $G^{\prime}$ with vertex set $\left\{v_{1}, \ldots, v_{t}\right\}$ so that $\left(v_{i}, v_{j}\right)$ is an edge in $G^{\prime}$ if and only if $F_{i} \cap F_{j} \neq \emptyset$.

In the following, $\left(\begin{array}{c}{[t]} \\ j\end{array}\right)$ is the set of $j$-subsets of $[t]$. We calculate

$$
\begin{gathered}
\beta_{j-1, j d-1}^{T}(I)=\left|\left\{\left(i_{1}, \ldots, i_{j}\right) \in\left(\begin{array}{c}
{[t]} \\
j
\end{array}\right): \sum_{p, q \in[j]}\left|F_{i_{p}} \cap F_{i_{q}}\right|=1\right\}\right| \leq \\
\left|\left\{\left(i_{1}, \ldots, i_{j}\right) \in\left(\begin{array}{c}
{[t]} \\
j
\end{array}\right):\left|\left\{\{p, q\} \in\left(\begin{array}{c}
{[j]} \\
2
\end{array}\right):\left(v_{i_{p}}, v_{i_{q}}\right) \in E\left(G^{\prime}\right)\right\}\right|=1\right\}\right| .
\end{gathered}
$$

Label the latter expression by $P_{j}\left(G^{\prime}\right) ; P_{j}\left(G^{\prime}\right)$ is the number of induced copies of a single edge on $j$ vertices in $G^{\prime}$. It suffices to show that $P_{j}\left(G^{\prime}\right) \leq\left(\begin{array}{c}t \\ j\end{array}\right)-\left(\begin{array}{c}t_{1} \\ j\end{array}\right)-\ldots-\left(\begin{array}{c}t_{j-1} \\ j\end{array}\right)$. We start with the case $j=3$ and then prove the theorem for general $j$.

With $\operatorname{deg} v$ denoting the degree of a vertex $v$ in $G^{\prime}$, and $a$ the average of $\operatorname{deg} v$ over all vertices $v$ in $G^{\prime}$, we have

$$
\begin{gathered}
P_{3}\left(G^{\prime}\right) \leq \frac{1}{2}\left|\left\{\left(v_{p}, v_{q}, v_{r}\right) \in V\left(G^{\prime}\right)^{3}:\left(v_{p}, v_{r}\right) \notin E\left(G^{\prime}\right),\left(v_{q}, v_{r}\right) \in E\left(G^{\prime}\right)\right\}\right|= \\
\frac{1}{2} \sum_{v \in V\left(G^{\prime}\right)}(\operatorname{deg} v)(t-1-\operatorname{deg} v) .
\end{gathered}
$$

The latter equality holds since for a vertex $v$, there are $\operatorname{deg} v$ vertices $v_{i}$ adjacent to $v$ in $G$, and there are $t-1-\operatorname{deg} v$ vertices not adjacent to $v$. Then $\beta_{2,3 d-1}^{T}(I) \leq \frac{1}{2} \operatorname{ta}(t-1-a)$, which follows from the inequality

$$
\sum_{v} \operatorname{deg}^{2} v \geq\left(\sum_{v} \operatorname{deg} v\right)^{2} / t=t a^{2} .
$$


We apply induction on $t$ with the base cases $P_{3}\left(G^{\prime}\right)=0$ for $t=1,2$ clear. First consider the case that $t=2 k$ is even. If $a \leq k-1$, then $a(2 k-1-a) \leq k(k-1)$ and so

$$
P_{3}\left(G^{\prime}\right) \leq \frac{1}{2} 2 k a(2 k-1-a) \leq k(k-1)(k)=\left(\begin{array}{c}
2 k \\
3
\end{array}\right)-\left(\begin{array}{c}
k \\
3
\end{array}\right)-\left(\begin{array}{l}
k \\
3
\end{array}\right) .
$$

Otherwise, if $a>k-1$, then there exists a vertex $v \in V\left(G^{\prime}\right)$ with $\operatorname{deg} v \geq k$. By the inductive hypothesis, $P_{3}\left(G^{\prime}-v\right) \leq\left(\begin{array}{c}2 k-1 \\ 3\end{array}\right)-\left(\begin{array}{c}k \\ 3\end{array}\right)-\left(\begin{array}{c}k-1 \\ 3\end{array}\right)$. Also $P_{3}\left(G^{\prime}\right)-P_{3}\left(G^{\prime}-v\right) \leq$ $\left(\begin{array}{c}2 k-1 \\ 2\end{array}\right)-\left(\begin{array}{c}k \\ 2\end{array}\right)$ since the induced subgraph on $v$ and vertices $u_{1}, u_{2}$ is not a single edge if $u_{1}$ and $u_{2}$ are both neighbors of $v$. Hence $P_{3}\left(G^{\prime}\right) \leq$

$$
\left(\left(\begin{array}{c}
2 k-1 \\
3
\end{array}\right)-\left(\begin{array}{l}
k \\
3
\end{array}\right)-\left(\begin{array}{c}
k-1 \\
3
\end{array}\right)\right)+\left(\left(\begin{array}{c}
2 k-1 \\
2
\end{array}\right)-\left(\begin{array}{l}
k \\
2
\end{array}\right)\right)<\left(\begin{array}{c}
2 k \\
3
\end{array}\right)-\left(\begin{array}{l}
k \\
3
\end{array}\right)-\left(\begin{array}{l}
k \\
3
\end{array}\right) .
$$

Now consider $t=2 k+1$. If $a \leq k-1$, then

$$
P_{3}\left(G^{\prime}\right) \leq \frac{1}{2}(2 k+1)(k-1)(k+1)<\left(\begin{array}{c}
2 k+1 \\
3
\end{array}\right)-\left(\begin{array}{c}
k+1 \\
3
\end{array}\right)-\left(\begin{array}{l}
k \\
3
\end{array}\right) .
$$

If $a>k-1$, then there exists $v \in V\left(G^{\prime}\right)$ with $\operatorname{deg} v \geq k$. By the inductive hypothesis, $P_{3}\left(G^{\prime}-v\right) \leq\left(\begin{array}{c}2 k \\ 3\end{array}\right)-\left(\begin{array}{c}k \\ 3\end{array}\right)-\left(\begin{array}{l}k \\ 3\end{array}\right)$. Also $P_{3}\left(G^{\prime}\right)-P_{3}\left(G^{\prime}-v\right) \leq\left(\begin{array}{c}2 k \\ 2\end{array}\right)-\left(\begin{array}{c}k \\ 2\end{array}\right)$. Hence

$$
P_{3}\left(G^{\prime}\right) \leq\left(\left(\begin{array}{c}
2 k \\
3
\end{array}\right)-\left(\begin{array}{l}
k \\
3
\end{array}\right)-\left(\begin{array}{l}
k \\
3
\end{array}\right)\right)+\left(\left(\begin{array}{c}
2 k \\
2
\end{array}\right)-\left(\begin{array}{l}
k \\
2
\end{array}\right)\right)=\left(\begin{array}{c}
2 k+1 \\
3
\end{array}\right)-\left(\begin{array}{c}
k+1 \\
3
\end{array}\right)-\left(\begin{array}{l}
k \\
3
\end{array}\right) .
$$

Now we complete the proof for general $j$. Choose $S \subset V\left(G^{\prime}\right)$ with $|S|=j$ and $v \in S$ for some vertex $v$. Then $G^{\prime}[S]$ is not a single edge if either of the following conditions holds: for all $v \neq u \in S, v$ and $u$ are adjacent, or for all $v \neq u \in S, v$ and $u$ are not adjacent and $G^{\prime}[S-v]$ is not a single edge. Note that these conditions are not exhaustive.

There are $\left(\begin{array}{c}\operatorname{deg} v \\ j-1\end{array}\right)$ ways in which the first condition above is satisfied, and by the inductive hypothesis, there are at least $\left(\begin{array}{c}t_{1}^{\prime} \\ j-1\end{array}\right)+\ldots+\left(\begin{array}{c}t_{j-2}^{\prime} \\ j-1\end{array}\right)$ ways in which the second condition is satisfied, where $\left(t_{1}^{\prime}, \ldots, t_{j-2}^{\prime}\right)$ is a nearly even $(j-2)$-partition of $t-\operatorname{deg} v-1$. Hence the number of induced subgraphs of $G^{\prime}$ that are not a single edge, contain $j$ vertices, and contain $v$ is at least $\left(\begin{array}{c}\operatorname{deg} v \\ j-1\end{array}\right)+\left(\begin{array}{c}t_{1}^{\prime} \\ j-1\end{array}\right)+\ldots+\left(\begin{array}{c}t_{j-2}^{\prime} \\ j-1\end{array}\right)$. Since $\left(\operatorname{deg} v, t_{1}^{\prime}, \ldots, t_{j-2}^{\prime}\right)$ is a partition of $t-1$, this quantity is bounded below by $\left(\begin{array}{c}\tilde{t}_{1} \\ j-1\end{array}\right)+\ldots+\left(\begin{array}{c}\tilde{t}_{j-1} \\ j-1\end{array}\right)$, where $\left(\tilde{t}_{1}, \ldots, \tilde{t}_{j-1}\right)$ is a nearly even $(j-1)$-partition of $t-1$.

Adding over all such $v, P_{j}\left(G^{\prime}\right) \leq$

$$
\frac{t}{j}\left(\left(\begin{array}{l}
t-1 \\
j-1
\end{array}\right)-\left(\begin{array}{c}
\tilde{t}_{1} \\
j-1
\end{array}\right)-\ldots-\left(\begin{array}{c}
\tilde{t}_{j-1} \\
j-1
\end{array}\right)\right) \leq\left(\begin{array}{l}
t \\
j
\end{array}\right)-\left(\begin{array}{c}
t_{1} \\
j
\end{array}\right)-\ldots-\left(\begin{array}{c}
t_{j-1} \\
j
\end{array}\right),
$$

where $\left(t_{1}, \ldots, t_{j-1}\right)$ is a nearly even $(j-1)$-partition of $t$. This prove the result.

For $j=3$, the upper bound of Theorem 4.1 is attained by the degree 2 hypergraph with vertices $u_{1}, u_{2}, v_{1}, \ldots, v_{t_{1}}, w_{1}, \ldots, w_{t_{2}}$ and edges

$$
\left\{\left(u_{1}, v_{1}\right) \ldots\left(u_{1}, v_{t_{1}}\right),\left(u_{2}, w_{1}\right) \ldots\left(u_{2}, w_{t_{2}}\right)\right\} .
$$


The bound does not seem to be tight for $j>3$.

What about other Betti numbers of the form $\beta_{2, \bullet}(I)$ ? A higher bound is necessary for $\beta_{2,6}(I)$ in the case that $d=3$. There exists a degree 3 squarefree monomial ideal $I$ on 6 generators with $\beta_{2,6}(I)=\left(\begin{array}{l}6 \\ 3\end{array}\right)$, namely

$$
I=\left(x_{1} x_{2} x_{4}, x_{1} x_{2} x_{5}, x_{1} x_{3} x_{6}, x_{1} x_{3} x_{7}, x_{2} x_{3} x_{8}, x_{2} x_{3} x_{9}\right) .
$$

It can also be seen by exhaustive search that $I$ is the only ideal, up to isomorphism, satisfying these properties, and that there does not exist a degree 3 ideal on 7 generators satisfying $\beta_{2,6}(I)=\left(\begin{array}{l}7 \\ 3\end{array}\right)$ or even $\beta_{2,6}^{T}=\left(\begin{array}{l}7 \\ 3\end{array}\right)$; that is, there is no degree 3 hypergraph with 7 edges such that all 3 -sets of edges $F_{1}, F_{2}, F_{3}$ satisfy $\left|F_{1} \cup F_{2} \cup F_{3}\right|=6$. It follows that, for a degree 3 squarefree monomial ideal $I$ on $t$ generators, $\beta_{2,6}^{T}(I) \leq\left(\begin{array}{l}t \\ 3\end{array}\right)-T(t, 7,3)$. Here $T(t, 7,3)$ is the Turán number, defined to be the minimum cardinality of a collection $W$ of 3 -subsets of $[t]$ such that every 7-subset of $[t]$ contains an element of $W$. See the reference paper [18] for more on Turán numbers.

In analogy with Theorem 4.1, we make the following conjecture.

Conjecture 4.2 Let I be a degree 3 squarefree monomial ideal with $t$ generators, and let $\left(t_{1}, t_{2}, t_{3}\right)$ be a nearly even 3 -partition of $t$. Then

$$
\beta_{2,6}(I) \leq\left(\begin{array}{l}
t \\
3
\end{array}\right)-\left(\begin{array}{c}
t_{1} \\
3
\end{array}\right)-\left(\begin{array}{c}
t_{2} \\
3
\end{array}\right)-\left(\begin{array}{c}
t_{3} \\
3
\end{array}\right) .
$$

If Conjecture 4.2 is true, then the bound is tight and is attained by the ideal on variables $x_{1}, x_{2}, x_{3}, y_{1}, \ldots, y_{t_{1}}, z_{1}, \ldots, z_{t_{2}}, w_{1}, \ldots, w_{t_{3}}$ given by

$$
I=\left(x_{1} x_{2} y_{1}, \ldots, x_{1} x_{2} y_{t_{1}}, x_{1} x_{3} z_{1}, \ldots, x_{1} x_{3} z_{t_{2}}, x_{2} x_{3} w_{1}, \ldots, x_{2} x_{3} w_{t_{3}}\right) .
$$

\section{Acknowledgments}

The author has been supported while working on this project by a graduate fellowship from VIGRE NSF Grant DMS-0354131 and from DMS-0801152. The author also wishes to thank the referee for many helpful comments.

\section{References}

[1] A. Bigatti, Upper bounds for the Betti numbers of a given Hilbert function, Comm. Algebra, 21 (1993), 2317-2334.

[2] M. Brun and T. Römer, Betti Numbers of $\mathbb{Z}^{n}$-Graded Modules, Comm. Algebra, 32 (2004), 4589-4599.

[3] H. Charalambous, Betti numbers of multigraded modules, J. Algebra, 137 (1991), 491-500. 
[4] A. Corso and U. Nagel, Monomial and toric ideals associated to Ferrers graphs, Trans. Amer. Math. Soc 361 (2009), 1371-1395.

[5] A. Corso and U. Nagel, Specializations of Ferrers ideals, J. Algebraic Combin. 28 (2008), 425-437.

[6] A. Duval, C. Klivans, and J. Martin, Simplicial matrix-tree theorems, to appear in Trans. Amer. Math. Soc.

[7] D. Eisenbud, M. Green, K. Hulek, S. Popescu, Restricting linear syzygies: algebra and geometry, Compositio Math., 141 (2005), 1460-1478.

[8] E. Evans and P. Griffith, Binomial behavior of Betti numbers for modules of finite length, Pacific J. Math, 133 (1988), 267-276.

[9] M. Goff, Bounding Betti numbers of bipartite graph ideals, J. of Pure and Appl. Algebra, to appear, math arXiv: 0807.1540.

[10] H.T. Hà, A. Van Tuyl, Monomial ideals, edge ideals of hypergraphs, and their graded Betti numbers, J. Algebr. Comb., 27 (2008), 215-245.

[11] H.T. Hà, A. Van Tuyl, Splittable ideals and the resolutions of monomial ideals, $J$. Algebra, 309 (2007), 405-425.

[12] H.T. Hà, A. Van Tuyl, Resolutions of squarefree monomial ideals via facet ideals: a survey, Contemp. Math., 448 (2007), 91-117.

[13] S. Jacques, Betti numbers of graph ideals, University of Sheffield, Ph.D. thesis, math arXiv: 0410107v1.

[14] S. Jacques, M. Katzman, The Betti numbers of forests, math arXiv: 0501226v2.

[15] M. Katzman, Characteristic-independence of Betti numbers of graph ideals, J. Combin. Theory Ser. A, 113 (2006), 435-454.

[16] E. Miller and B. Sturmfels, Combinatorial Commutative Algebra, Springer Science+Business Media, Inc., 2005.

[17] U. Nagel and V. Reiner, Betti numbers of monomial ideals and shifted skew shapes, Electron. J. Combin., 16 (2009) \#R3.

[18] A. Sidorenko, What we know and what we do not know about Turán numbers, Graphs Combin., 11 (1995), 179-199.

[19] E. Spanier, Algebraic Topology, Springer-Verlag New York, Inc., 1966.

[20] R. Stanley, Combinatorics and Commutative Algebra, Second Edition, Birkhäuser, Boston, 1996.

[21] R.H. Villarreal, Cohen-Macaulay graphs, Manuscripta Math., 109 (1990), 159-174. 